\title{
Eradication of Helicobacter pylori infection in patients with duodenal ulcer and non-ulcer dyspepsia and analysis of one-year reinfection rates
}

\author{
E. Della Libera, M.R.S. Rohr, \\ M. Moraes, E.S. Siqueira \\ and A.P. Ferrari Jr.
}

\author{
Unidade de Endoscopia, Divisão de Gastroenterologia, \\ Escola Paulista de M edicina, Universidade Federal de São Paulo, \\ São Paulo, SP, Brasil
}

\begin{abstract}
Correspondence

A.P. Ferrari Jr.

R. Pedro de Toledo, 980, Cj 66

04039-002 São Paulo, SP

Brasil

Fax: + 55-11-549-5699/573-9838

E-mail: angelo@ gastro.epm.br

Research partially supported by FAPESP (No. 95/3685-4).

Received December 21, 1999

Accepted March 6, 2001

Helicobacter pylori (HP) infection is endemic worldwide. The proposed treatment is expensive and there are few reports regarding reinfection rates in Brazil. The aim of this study was to compare the eradication rates obtained with two therapeutic options and to evaluate reinfection one year after treatment. This was a prospective randomized trial with 55 patients. Thirty-nine patients had active duodenal ulcer (DU) and 16 non-ulcer dyspepsia (NUD), and all tested positive for HP. Diagnosis was based on at least two positive tests: ultrarapid urease test, histology and/or culture. Patients were randomized to two groups: group OMC treated with $40 \mathrm{mg}$ omeprazole (once a day), 500 $\mathrm{mg}$ metronidazole and $250 \mathrm{mg}$ clarithromycin (twice daily) for 7 days, or group NA treated with $300 \mathrm{mg}$ nizatidine (once a day) and $1000 \mathrm{mg}$ amoxicillin (twice daily) for 14 days. Those patients in whom HP was eradicated were followed up for one year to evaluate reinfection. Twenty-five patients were randomized for OMC and 30 for NA. HP eradication occurred in $20 / 25$ patients $(80 \%)$ treated with OMC and $13 / 30(43 \%)$ treated with NA $(P=0.01)$. After reallocation because of initial treatment failure, the overall eradication rate was $44 / 51$ patients (86\%). After an average follow-up of one year, we evaluated 34 patients ( 23 with DU and 11 with NUD). Reinfection occurred in 3/34 patients $(7.6 \%)$. We conclude that OMC is effective for HP eradication, and that NA should not be used. Reinfection occurs in $7.6 \%$ of the patients in the first year after eradication.
\end{abstract}

Since the first description of Helicobacter pylori (HP) in gastric biopsies by Warren and Marshall (1), interest in gastric bacteriology was renewed. The infection is currently endemic worldwide with a high prevalence (up to $60 \%$ ) in developing regions such as South America, Africa and Asia (2-4).

Several pathogenic mechanisms have already been demonstrated (5). Infection is
Key words

- Helicobacter pylori

- Peptic disease

- Non-ulcer dyspepsia

- Gastritis associated with gastric mucosa inflammation, even in the absence of duodenal ulcer (DU). Probably different responses depend on interactions between the bacteria and host factors (6). H. pylori infection causes chronic gastritis, gastric and duodenal ulcer, probably gastric adenocarcinoma $(7,8)$ and mucosa-associated lymphoid tissue (9). The role of HP in non-ulcer dyspepsia (NUD) is contro- 
versial. A variety of tests have been proposed for infection diagnosis: urease test, histology, culture and urea breathing test $(10,11)$, all with high sensitivity and specificity.

Therapeutic studies using bismuth, amoxicillin and metronidazole have reported up to $90 \%$ effectiveness (6) but with the disadvantage of poor patient compliance. Therapy with amoxicillin associated with omeprazole was reported to eradicate infection in more than $80 \%$ of patients, with lower side effect rates and cost (12). The recurrence rate after HP eradication in adult patients in Brazil is still unknown. In developed countries, where prevalence is lower, recurrence ranges from 1 to $5 \%$ one year after treatment (13).

The present study was a blind prospective randomized trial, performed between March 1996 and April 1998, including patients referred to the Endoscopy Unit, Division of Gastroenterology, UNIFESP-EPM, because of dyspepsia. The study was approved by the Ethics Committee of Hospital São Paulo, UNIFESP-EPM. Written consent was obtained from all patients.

Inclusion criteria were age between 18 and 70 years and at least two HP-positive tests. Group 1 consisted of patients with active DU and group 2 of NUD patients (normal or nonerosive gastritis on endoscopy, and normal abdominal ultrasound and stool examination). Exclusion criteria were severe chronic diseases that hindered the proposed therapy, pregnancy and/or breastfeeding, use of antibiotics, bismuth or nonsteroidal anti-inflammatory drugs in the 4 weeks preceding index endoscopy, and a previous history of side effects to the drugs proposed in the study. In the DU group we excluded patients with gastric ulcer, neoplasia, previous gastric surgery or complicated DU (bleeding or stricture). In the NUD group, patients with diagnosed worm infestation or ultrasound abnormalities (gallstones, bile duct stones, pancreatic diseases), hiatus hernia, esophagitis, or erosive gastritis were excluded.
The diagnosis of HP infection was based on five antral biopsies, two for the ultrarapid urease test (10), two for histology and one for culture. Histology slides were stained with Giemsa. One specimen was immediately placed on an HP-specific growth medium. The plates were incubated under microaerobiosis and examined after 4 to 7 days. $H$. pylori infection was considered to be present when at least two tests were positive and absent when at least two tests were negative. Table 1 shows sensitivity, specificity, positive and negative predictive values and accuracy for all tests.

Patients in the NUD group answered a questionnaire to evaluate the frequency, intensity and duration of five symptoms: epigastric pain, night pain, gastric fullness, heartburn, and eructation, all graded as light, moderate or severe.

We reported good results in eradicating HP infection with amoxicillin monotherapy (14). In an attempt to evaluate an easier and cheaper therapeutic option, patients were randomized to two treatment groups as follows: group OMC - $40 \mathrm{mg}$ omeprazole (once a day), $500 \mathrm{mg}$ metronidazole and $250 \mathrm{mg}$ clarithromycin (twice daily) for 7 days; group NA - $300 \mathrm{mg}$ nizatidine (once a day) and $1000 \mathrm{mg}$ amoxicillin (twice daily) for 14 days. All drugs were supplied free of charge. Patients were instructed to bring back the containers and medications for compliance evaluation and did not receive any maintenance treatment. Those who took less than $50 \%$ of the supplied drugs were considered violators and excluded from the study and statistical analysis. Clinical improvement, ulcer healing and HP eradication were evaluated blindly.

Follow-up consisted of clinical visits after 30 days, 6 months and 1 year or whenever necessary. Endoscopy was performed 60 days after the end of treatment to evaluate eradication and ulcer healing. Patients with eradicated infection were followed up to one year and then submitted to another endoscopy for 
evaluation of ulcer relapse and HP status. Non-ulcer patients with failed eradication were referred to our outpatient clinic. Ulcer patients with failed eradication were crossed over to the other therapeutic group and again evaluated after 60 days by endoscopy and HP tests. Patients whose infection was eradicated were submitted to endoscopy one year later. Those who were still HP carriers were considered treatment failures and referred to our outpatient clinic.

Sixty-two patients were included: 39 with active DU (group 1) and 23 with NUD (group 2). Median age was 32.8 years (range from 18 to 66 years), with 27 men and 35 women. Seven patients were excluded before randomization: 6 did not show up for the second clinical visit (to receive the drugs) and 1 patient had symptom worsening in the week between the first endoscopy and randomization. He came back to the Endoscopy Unit and a second procedure revealed erosive gastritis, probably secondary to nonsteroidal anti-inflammatory drugs, and he was not randomized.

The OMC group consisted of 25 patients (19 with DU and 6 with NUD) and the NA group consisted of 30 patients (20 with DU and 10 with NUD). H. pylori eradication was achieved in $20 / 24$ patients $(83 \%)$ with OMC and in $13 / 30$ patients $(43 \%)$ with NA (P = $0.007)$. One patient was excluded because of protocol violation. Based on an intention to treat analysis, eradication occurred in 20/25 patients $(80 \%)$ and $13 / 30$ patients $(43 \%)$ in the OMC and NA groups, respectively $(\mathrm{P}=$ $0.01)$.

When ulcer and non-ulcer patients were analyzed separately, OMC led to HP eradication in $83 \%$ of patients in both groups. Among non-ulcer patients, only two reported symptomatic improvement, two had no changes and one patient reported worsening.

Treatment with NA led to HP eradication in $7 / 20(35 \%)$ DU and 6/10 (60\%) NUD patients. Thirteen patients with DU were still infected after treatment: 11 were treated with OMC and eradication was achieved in 9/11 patient (82\%). Two patients were excluded at this point due to protocol violation. Among the 6 patients with NUD in whom HP was eradicated, there was symptomatic improvement in 4, and symptoms remained unchanged in 2. Table 2 and Figure 1 show the results of HP eradication for all patients.

Overall, 36/39 patients (92\%) with DU obtained complete healing and 3 (8\%) continued to have an active lesion and were referred to our outpatient clinic. Symptom scores improved in $6 / 11$ patients (54\%) with NUD in whom HP was successfully treated. Considering both treatment arms, HP infection was eradicated in $44 / 51$ patients $(84 \%)$ in a per protocol analysis and 44/55 (80\%) if an intention to treat approach was considered.

Table 1. Results of tests for the diagnosis and therapy monitoring of Helicobacter pylori infection.

\begin{tabular}{lcrrrr}
\hline Test & S & E & PPV & NPV & AC \\
\hline Urease & 96.77 & 98.18 & 98.36 & 96.42 & 97.43 \\
Culture & 91.52 & 100.00 & 100.00 & 91.37 & 95.53 \\
Histology & 98.38 & 89.08 & 91.04 & 98.00 & 94.01 \\
\hline
\end{tabular}

Data are reported as \%. S = sensitivity; $\mathrm{E}=$ specificity; PPV = positive predictive value; NPV $=$ negative predictive value; $A C=$ accuracy.

Table 2. Helicobacter pylori (HP) eradication and ulcer healing after the first treatment or after reallocation.

\begin{tabular}{lcccc}
\hline Treatment & Group & N & HP eradication (PP) & DU healing \\
\hline Initial & OMC & DU (19) & $15 / 18(83 \%)^{\mathrm{a}}$ & $17 / 19(89 \%)$ \\
& & NUD (6) & $5 / 6(83 \%)$ & - \\
& & Total (25) & $20 / 24(83 \%)$ & $17 / 19(89 \%)$ \\
& NA & DU (20) & $7 / 20(35 \%)$ & $17 / 20(85 \%)$ \\
& & NUD (10) & $6 / 10(60 \%)$ & - \\
Reallocation & Total (30) & $13 / 30(43 \%)$ & $17 / 20(85 \%)$ \\
& OMC & DU (13) & $9 / 11(82 \%)^{\mathrm{b}}$ & $10 / 13(77 \%)$ \\
& NA & DU (2) & $2 / 2(100 \%)$ & $1 / 2(50 \%)$
\end{tabular}

a One patient was excluded because of protocol violation; btwo patients were excluded because of protocol violation. PP: per protocol analysis; DU: duodenal ulcer; NUD: non-ulcer dyspepsia; OMC: omeprazole, metronidazole and clarithromycin; NA: nizatidine and amoxicillin; N: number of patients in each group. 
Figure 1. Flow chart of all patients. *From the 62 patients, six were excluded before randomization because they did not show up for a clinical visit, and one was excluded because his symptoms worsened during the week before randomization. For abbreviations, see legend to Table 2.
One year after eradication $(12.47 \pm 4.56$ months) we were able to evaluate 34 patients (23 DU and 11 NUD) for reinfection. The reinfection rate was $7.6 \%$ (3/34 patients), with all 3 patients being from the DU group. All DU patients presented healed lesions at the one-year control endoscopy, including the 3 with reinfection.

In patients with peptic ulcer, the importance of bacterial eradication has been well established, with reported high recurrence rate in those who remain HP positive. In Brazil, the cost of triple therapy and patient compliance are major problems regarding HP treatment. A previous study using omeprazole and amoxicillin to treat patients with HP-associated ulcers demonstrated good clinical results and high eradication rates (12), but these results have not been reproduced in Brazil.

There is no consensus about the role of HP in NUD. Functional dyspepsia is defined as epigastric pain or chronic discomfort (15), a definition including a series of symptoms like pain, early satiety, nausea, anorexia, heartburn, and regurgitation. Identification of HP has led to speculation regarding its correlation with functional dyspepsia and therefore bacterial eradication would contribute to clinical improvement in such patients. Several studies have produced contradictory results. McCarthy et al. (16) studied 83 patients with functional dyspepsia and HP and reported significantly impaired symptoms in untreated patients or those with reinfection.

The treatment used in our study (OMC) showed an eradication rate of $83 \%$ in a per protocol analysis and $80 \%$ in an intention to treat approach. Side effects were minor and no patient interrupted treatment because of them. All patients excluded from the OMC group were excluded due to protocol violation, denoting difficulty in dealing with the large number of tablets, since the problem of cost was eliminated. Previous use of amoxicillin did not interfere with the response to OMC: patients treated in this group after failure of NA showed a similar eradication rate $(82 \%)$. Development of resistance to amoxicillin is not common $(13,17)$. The eradication of HP with a 7-day course of OMC was similar to that obtained in 14-day therapeutic trials previously reported $(18,19)$. In

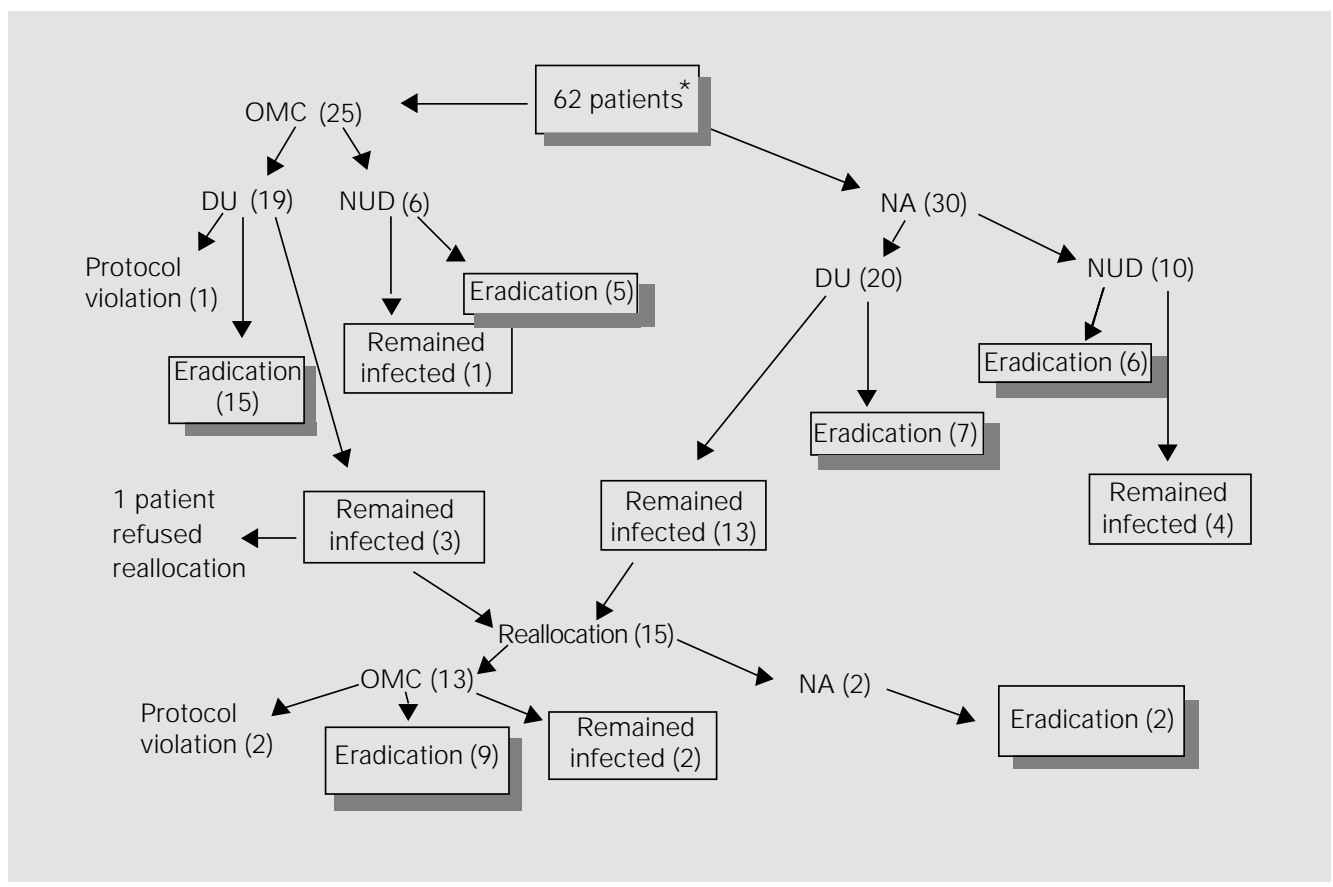


our opinion, the 7-day treatment has some advantages, namely lower cost and probably better compliance, and most authors consider it the best first choice.

Patients treated with NA for 14 days presented a low eradication rate $(43 \%)$, confirming that therapy with only one antibiotic should not be prescribed. Among patients treated with NA the eradication rate was higher in the NUD group (60\%) when compared to the ulcer group (35\%).

Among the 11 patients with NUD in whom the infection was eradicated, 6/11 (55\%) presented symptomatic improvement, in $4 / 11(36 \%)$ the symptoms were unchanged and $1 / 11(9 \%)$ presented clinical worsening. Therefore, HP eradication was not associated with clinical improvement and the role of reinfection was not assessed in this group of patients.

The reinfection rate after one year was $7.6 \%$ in our study. This is higher than in developed areas like the USA and Europe, where reinfection rates range from 1 to $5 \%$ (13), probably because of low socioeconomic status. However, we should consider the loss of patient follow-up in this phase of the study. It is possible that, although already higher than in other reports, this rate represents an underestimated value.

We conclude that 7-day treatment with omeprazole, metronidazole and clarithromycin is effective in the eradication of $H$. pylori (83\%). Although cheaper, monotherapy with amoxicillin should not be used due to the low eradication rate obtained. Reinfection occurred in $7.6 \%$ of the patients after an average follow-up of 12 months.

\section{References}

1. Warren J R \& Marshall B (1983). Unidentified curved bacilli on gastric epithelium in active chronic gastritis. Lancet, 1: 12731275.

2. Taylor DN \& Blaser MJ (1991). The epidemiology of Helicobacter pylori infection. Epidemiologic Reviews, 13: 42-59.

3. Holambe O, Omotara BA, Eldridge J \& J ones DM (1992). Helicobacter pylori, the most common bacterial infection in Africa: a random serological study. American J ournal of Gastroenterology, 87: 2830.

4. Megraud F, Rabbé MPB, Denis F, Belbouri A \& Hoa DQ (1989). Seroepidemiology of Campylobacter pylori infection in various populations. J ournal of Clinical Microbiology, 27: 1870-1873.

5. Calam J , Gibbons A, Healey ZV, Bliss P \& Arebi N (1997). How does Helicobacter pylori cause mucosal damage? Its effect on acid and gastrin physiology. Gastroenterology, 113 (Suppl 6): S43-S49.

6. Tytgat GNJ, Noach LA \& Rauws EAJ (1993). Helicobacter infection and duodenal ulcer disease. Gastroenterology Clinics of North America, 22: 127-139.

7. Graham DY, Go MF \& Genta RM (1995). Helicobacter pylori, duodenal ulcer, gastric cancer: Tunnel vision or blinders? Annals of Medicine, 27: 589-594.

8. Graham DY (1989). Campylobacter pylori and peptic ulcer disease. Gastroenterology, 96: 615-625.

9. Roggero E, Zucca E, Pinotti MD, Pascarella $A$, Capella $C$, Savio $A$, Pedrinis $E$, Paterlini MD, Venco A \& Cavalli F (1995). Eradication of Helicobacter pylori infection in primary low-grade gastric lymphoma of mucosa-associated lymphoid tissue. Annals of Internal Medicine, 122: 767-769.

10. Thillainayagam AV, Arvind AS, Cook RS, Harrison IG, Tabaqchali S \& Farthing MJ G (1991). Diagnostic efficiency of an ultrarapid endoscopy room test for Helicobacter pylori. Gut, 32: 467-469.

11. Loffeld RJ , Stobberingh E \& Arends J W (1993). A review of diagnostic techniques for Helicobacter pylori infection. Digestive Diseases, 11: 173-180.

12. Bayerdorffer $\mathrm{E}$, Miehlke $\mathrm{S}, \mathrm{Mannes} \mathrm{GA}$, Sommer A, Hochter W, Weingart J, Heldwein W, Klann H, Simon T, Schmitt W, Bastlein E, Eimiller A, Hatz R, Lehn N, Dirschedl P \& Stolte M (1995). Doubleblind trial of omeprazole and amoxicillin to cure Helicobacter pylori infection in patients with duodenal ulcer. Gastroenterology, 108: 1412-1417.

13. Walsh JH \& Peterson WL (1995). The treatment of Helicobacter pylori in the management of peptic ulcer disease. New England J ournal of Medicine, 333:
984-991.

14. Ferrari J r AP, Geocze S, Lanzoni VPB, Toledo MRF, Trabulsi LR \& Vilela MP (1991). Use of ampicillin plus ranitidine for Helicobacter pylori gastritis. Brazilian J ournal of Medical and Biological Research, 24: 567572.

15. Heading RC (1991). Definitions of dyspepsia. Scandinavian J ournal of Gastroenterology, 26 (Suppl 182): S1-S6.

16. McCarthy $C$, Patchett $S$, Collins RM, Beattie S, Keane C \& O'M orain C (1995). Long-term prospective study of Helicobacter pylori in nonulcer dyspepsia. Digestive Diseases and Sciences, 40: 114119.

17. Marshall BJ (1994). Helicobacter pylori. American J ournal of Gastroenterology, 89 (Suppl 8): S116-S128.

18. Yousfi MM, el-Zimaity HM, al-Assi MT, Cole RA, Genta RM \& Graham DY (1995). Metronidazole, omeprazole, and clarithromycin: an effective combination therapy for Helicobacter pylori infection. Alimentary Pharmacology and Therapeutics, 9: 209-212.

19. Chiba N (1996). Omeprazole and clarithromycin with and without metronidazole for the eradication of Helicobacter pylori. American J ournal of Gastroenterology, 91: 2139-2143. 